Check for updates

Cite this: RSC Adv., 2017, 7, 49144

Received 16th September 2017 Accepted 15th October 2017

DOI: 10.1039/c7ra10294a

rsc.li/rsc-advances

\section{Enhanced performance of perovskite solar cells by strengthening a self-embedded solvent annealing effect in perovskite precursor films $\uparrow$}

\author{
Xiaobing Cao, ${ }^{a}$ Lili Zhi, ${ }^{\mathrm{b}}$ Yahui $\mathrm{Li}^{\mathrm{a}}{ }^{\text {Xian Cui, }}{ }^{\mathrm{a}}$ Lijie Ci, (D) ${ }^{\mathrm{b}}$ Kongxian Ding ${ }^{\mathrm{c}}$ \\ and Jinquan Wei $\mathbb{D}$ *a
}

\begin{abstract}
The solvent embedded in the intermediate phase is widely observed in the fabrication of perovskite films. The perovskite precursor films obtained from Lewis adducts through molecular exchange contain some residue solvent. It has an intrinsic solvent annealing effect during the annealing process. Here, we predeposit a protective layer on the perovskite precursor films to retard the escape of solvent during the annealing process. The restricted solvent strengthens the solvent annealing effect during the formation of perovskite films. As a result, the perovskite quality, including grain size and crystallization, is improved significantly, which leads to efficient charge transportation, low recombination rate, and enhancement of the photovoltaic performance of the corresponding perovskite solar cells.
\end{abstract}

\section{Introduction}

The efficiency of perovskite solar cells (PSCs) has been boosted from an initial $3.8 \%$ to $22.1 \%$ in recent years. ${ }^{1,2}$ The most important approach to obtain highly efficient PSCs is to improve the quality of the light harvest layer in PSCs, including improving morphology, ${ }^{3,4}$ increasing perovskite grain size, ${ }^{5,6}$ improving crystallization ${ }^{7,8}$ and optimizing composition. ${ }^{9}$ Among all these approaches, we need basically to improve the photovoltaic performance by increasing the perovskite grain size. The large perovskite grains can reduce trap density, which is beneficial to improve the performance ${ }^{\mathbf{1 0}}$ and stability ${ }^{\mathbf{1 1}}$ of the perovskite solar cells.

Up to now, various methods have been proposed to fabricate smooth perovskite films with large grains. For example, Nie et al. reported a solution-based hot-cast technique to grow high quality perovskite film with millimeter-scale crystalline grains. ${ }^{12}$ Xiao et al. adopted a fast deposition-crystallization processing for obtaining uniform perovskite films with large grains. ${ }^{13}$ Yang et al. developed a facile method to obtain large grain of $\mathrm{CH}_{3}$ $\mathrm{NH}_{3} \mathrm{I}_{3-x} \mathrm{Br}_{x}$ via an Ostwald ripening process by a simple $\mathrm{MABr}$ solution treatment. ${ }^{\mathbf{1 4}}$ Different additives were added into precursor solutions to increase perovskite grains. ${ }^{15,16}$ Especially,

${ }^{a}$ State Key Lab of New Ceramic and Fine Processing, School of Materials Science and Engineering, Tsinghua University, Beijing 100084, P. R. China. E-mail: jqwei@ tsinghua.edu.cn

${ }^{b}$ School of Materials Science and Engineering, Shandong University, Jinan 250061, Shandong, P. R. China

${ }^{c}$ Shenzhen Jiawei Solar Lighting Co., Ltd., Fuping Road, New Industrial Zone No. 1-4, Longgang District, Shenzhen 518112, Guangdong, P. R. China

$\dagger$ Electronic supplementary information (ESI) available. See DOI: 10.1039/c7ra10294a
Huang's group reported a novel solvent annealing to obtain large perovskite grains with excellent crystallinity. ${ }^{17}$ Typically, the solvent annealing process is as following: the perovskite film is exposed to different solvent (e.g., DMF, DMSO) in a Petri dish by optimizing annealing time and temperature. ${ }^{18,19}$ This process can promote the formation of large perovskite grains with excellent crystallization. However, the experiment condition for solvent annealing, such as the amount and distribution of solvent, annealing time, annealing temperature are extremely strict. The strict experiment conditions make it is difficult to fabricate high quality perovskite films with good reproducibility. ${ }^{20}$

The perovskite films fabricated through solution-based processing are usually intermediate phases contained some organic solvent molecules, and then the intermediate phases transform to perovskite film by removing solvent through annealing treatment. It has been a widely accepted fact that solvent embedded in intermediate phase play a key role in retarding the rapid reaction between $\mathrm{PbI}_{2}$ and $\mathrm{MAI}$ during the formation of high quality perovskite films. ${ }^{21-23}$ In our previous work, we fabricated smooth perovskite films from Lewis adducts through molecular exchange through a modified twostep method. ${ }^{24}$ The smooth perovskite films were ascribed to the solvent modulated the formation of perovskite film during molecular exchange and annealing process. ${ }^{25}$ It is solvent included perovskite precursor film, which is the product obtained after molecule exchange between solvent and MAI. We believe that the solvent molecule embedded in the perovskite precursor film may induce weak solvent annealing during annealing.

Here, we make use of the solvent embedded in the perovskite precursor films to induce an obvious solvent annealing effect. In 
order to retard the escape of the solvent, we pre-deposit a protective layer onto the perovskite precursor film before annealing. The restrained solvent strengthens the solvent annealing effect during annealing process, resulting in formation of smooth perovskite film with large grains.

\section{Experimental}

\section{Fabrication of the devices}

The clean of FTO substrates, deposition of $\mathrm{TiO}_{2}$ blocking layers, and $\mathrm{TiO}_{2}$ mesoporous layers have been described in details in our previous report. ${ }^{24}$ Perovskite films were fabricated through a modified two-step method..$^{24}$ A layer of $\mathrm{PbI}_{2}$ precursor film was firstly spin-coated from $\mathrm{PbI}_{2} / \mathrm{DMF}(1.4 \mathrm{M})$ solution at $5500 \mathrm{rpm}$ for $30 \mathrm{~s}$, and then dipped into a solution of $\mathrm{CH}_{3} \mathrm{NH}_{3} \mathrm{I}(\mathrm{MAI}) / 2$ propanol with concentration $30 \mathrm{mg} \mathrm{mL}^{-1}$ for $60 \mathrm{~s}$ to prepare perovskite precursor film. Here, we employ two different annealing approach on perovskite precursor film: traditional annealing approach without protective layer and sealed-style annealing approach with a protective layer onto perovskite precursor film. In traditional annealing approach, the perovskite precursor film was directly annealed at $100{ }^{\circ} \mathrm{C}$ for $120 \mathrm{~min}$ to obtain perovskite film. In the sealed-style annealing approach, a $\mathrm{PC}_{61} \mathrm{BM}$ protective layer was deposited on the top of perovskite precursor film, and then annealed at $100{ }^{\circ} \mathrm{C}$ for 120 min to obtain perovskite film. The $\mathrm{PC}_{61} \mathrm{BM}$ protective layer was washed by chlorobenzene several times before depositing a hole transportation layer. The hole transportation layer was deposited by spin-coating from HTL solution (100 mg SpiroOMeTAD, $40 \mu \mathrm{L}$ 4-tert-butylpyridine $(t \mathrm{BP}), 36.3 \mu \mathrm{L}\left(520 \mathrm{mg} \mathrm{mL} \mathrm{m}^{-1}\right.$ TFSI in acetonitrile) and $60 \mu \mathrm{L}$ FK102 (300 $\mathrm{mg} \mathrm{mL}^{-1}$ in acetonitrile) in $1 \mathrm{~mL}$ chlorobenzene). Finally, a gold electrode was deposited on HTL to form a complete perovskite solar cell. The active area of the electrode was fixed at $0.06 \mathrm{~cm}^{2}$.

\section{Characterization}

The perovskite films were characterized by field-emission scanning electron microscopy (SEM, MERLIN VP Compact), atomic force microscopy (AFM5500, Agilent), X-ray diffraction (XRD, D8-Advance), and UV/Vis absorption spectrometer (Cary 5000 UV-vis-NIR, Agilent Technologies) in a range from 400 to $900 \mathrm{~nm}$. Impedance spectra were measured by using an electrochemical workstation (CHI660D) in dark under a bias voltage of $0.9 \mathrm{~V}$ and an alternative signal of $10 \mathrm{mV}$ in frequency range from $1 \mathrm{~Hz}$ to $10^{6} \mathrm{~Hz}$. Steady photoluminescence (PL) spectra were measured by an Edinburgh FLS 920 instrument (Livingston, UK) excited by monochromatic xenon lamp source (central wavelength $\lambda=460 \mathrm{~nm}$ ). Time-resolved PL spectra were measured by a time-correlated single photon counting (TCSPC) system. The samples were excited by a $405 \mathrm{~nm}$ laser beam (EPL405). The photovoltaic performance of PSCs were measured by using a solar simulator (AM 1.5G, $100 \mathrm{~mW} \mathrm{~cm}^{-2}$, 91195, Newport). The obtained $J-V$ curves was recorded by Keithley 4200SCS parameter analyzer sweeping from -0.1 to $1.2 \mathrm{~V}$ at a scan rate of $5 \mathrm{mV} \mathrm{s}^{-1}$.

\section{Results and discussion}

Fig. 1 shows an illustration of the fabrication of perovskite by different annealing approaches. It forms Lewis adducts of $\mathrm{PbI}_{2} \cdot x \mathrm{DMF}$ when the $\mathrm{PbI}_{2} / \mathrm{DMF}$ solutions was spin-coated onto FTO substrates coated with $\mathrm{TiO}_{2}$ layer. It forms perovskite precursor of $\mathrm{MAI}-\mathrm{PbI}_{2}-\mathrm{DMF}$ when the Lewis adducts were dipped into MAI/IPA solution via molecular exchange. Fig. 1b shows the evolution of XRD curves during the formation of perovskite film. There are two obvious characteristic peaks located at $9.03^{\circ}, 9.56^{\circ}$ at low angle in $\mathrm{XRD}$ curves of $\mathrm{PbI}_{2} \cdot x \mathrm{DMF}$ (red line in Fig. 1b), corresponding to the plane (011) and (020) of the $\mathrm{PbI}_{2} \cdot x \mathrm{DMF} .{ }^{26}$ When $\mathrm{PbI}_{2} \cdot x \mathrm{DMF}$ film is dipped into MAI/ IPA solution, it forms perovskite precursor film of $\mathrm{MAI}-\mathrm{PbI}_{2}-$ $\mathrm{DMF}$, which is characterized by three characteristic peaks located between $5^{\circ}$ and $10^{\circ}$ in the XRD curve (see blue line in Fig. 1b). ${ }^{27,28}$ The MAI-PbI 2 -DMF film then transfer to $\mathrm{MAPbI}_{3}$ by removing the solvent through different annealing approaches. In the traditional annealing approach without protective layer, the perovskite intermediate films are directly annealed at $100{ }^{\circ} \mathrm{C}$ for $120 \mathrm{~min}$ (see Fig. 1c); while in the sealed-style annealing approach, perovskite intermediate films are covered with a protective layer, and then annealed at $100{ }^{\circ} \mathrm{C}$ for $120 \mathrm{~min}$. In previous work, it has been reported that $\mathrm{PC}_{61} \mathrm{BM}$ may penetrate into grain boundary for suppression of hysteresis of device. ${ }^{29}$ So we choose $\mathrm{PC}_{61} \mathrm{BM}$ as protective layer to study the novel annealing approach on the quality of perovskite film. The $\mathrm{PC}_{61} \mathrm{BM}$ is removed by washing in chlorobenzene solvent for several times (see Fig. 1d).

Fig. 2 is SEM images of the perovskite films annealed with and without protective layer. It is clear that the grains size strongly depends on the annealing approach. The average grains size increases from $206 \mathrm{~nm}$ to $351 \mathrm{~nm}$ (see Fig. S1†), and the average roughness of perovskite film also decrease from $20.3 \mathrm{~nm}$ to $13.3 \mathrm{~nm}$ (see Fig. $\mathrm{S} 2 \dagger$ ), when the perovskite precursor films are covered with a protective layer. Fig. $2 \mathrm{~b}$ and d are crosssectional SEM images of the perovskite film fabricated from different annealing approaches. The perovskite capping layers exhibit smooth and hole-free morphology with thickness of $\sim 340 \mathrm{~nm}$. The perovskite films contain few grain boundaries in the thickness direction fabricated by annealing without $\mathrm{PC}_{61} \mathrm{BM}$ protective layer, which is ascribed to the small grain size than the perovskite film thickness. However, the perovskite films fabricated by annealing with a protective layer exhibit columnlike structure. It contains only one grain in most area, which ascribes to the larger grains size than the film thickness. The column-like structure of perovskite film are extremely desired for high efficient PSCs due to reducing non-radiative charge carrier recombination at grain boundaries. ${ }^{30}$

Fig. 2e shows XRD curves of the perovskite films annealed with and without a $\mathrm{PC}_{61} \mathrm{BM}$ protective layer. All of the XRD curves exhibit several peaks located at $14.3^{\circ}, 28.6^{\circ}$, and $32.0^{\circ}$, which correspond to the (110), (220) and (310) planes of the $\mathrm{MAPbI}_{3}$, respectively. ${ }^{18}$ It is noted that the perovskite film annealed with a protective layer exhibits higher crystallization. We introduce intensity ratios of $I_{110} / I_{310}$ and $I_{220} / I_{310}$ to evaluate 
(a)

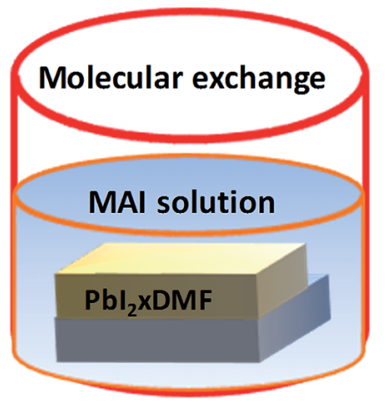

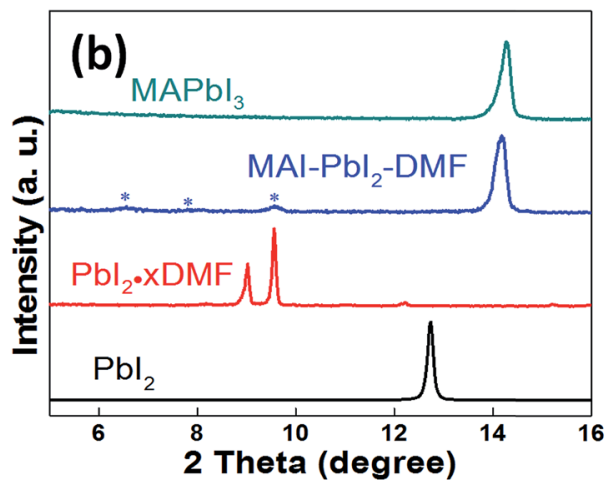

(c)

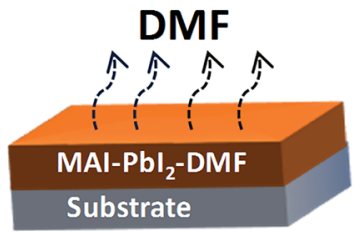

Without protective layer

(d)

Large grains

protective layer

MAI-Pbl ${ }_{2}$-DMF

Substrate

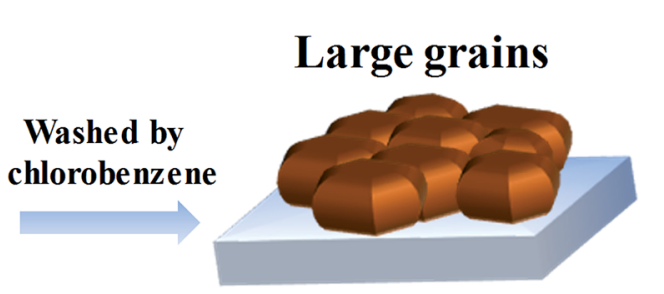

With protective layer

Fig. 1 Schematic illustration of the fabrication of perovskite films. (a) Molecular exchange process, (b) evolution of XRD patterns for different films during the formation of perovskite. Different approaches to fabricate perovskite films: (c) without protective layer, (d) with protective layer.

the grain orientation, which denoted as the intensity of (110) and (220) peaks divided by the intensity of (310) peak, respectively. ${ }^{31}$ The value of $I_{110} / I_{310}$ and $I_{220} / I_{310}$ of the perovskite film annealed with a protective layer are 7.53 are 3.54 , respectively. For comparison, the corresponding value are only 5.38 and 2.58 for the perovskite annealed without a protective layer. The increase of $I_{110} / I_{310}$ and $I_{220} / I_{310}$ indicate that the perovskite grains have a preferred orientation of (110), which is beneficial for the transportation of the carries. ${ }^{31}$ Fig. $2 \mathrm{f}$ shows UV absorption spectra of the perovskite films. The films show an onset at $775 \mathrm{~nm}$, corresponding to the bandgap of $\mathrm{MAPbI}_{3}$ $(\sim 1.55 \mathrm{eV}) .{ }^{32}$ The perovskite film annealed with protective layer exhibits stronger light absorption ability, especially in range from 400 to $550 \mathrm{~nm}$.

The improved crystallization and large grains size of $\mathrm{MAPbI}_{3}$ film can be ascribed to solvent annealing effect caused by DMF molecule embedded in perovskite precursor films. Without protective layer, DMF escape and evaporate from the MAI- $\mathrm{PbI}_{2}{ }^{-}$ DMF film easily during annealing process due to its low boiling point $\left(152{ }^{\circ} \mathrm{C}\right)^{33}$ and weak interaction between $\mathrm{Pb}^{2+}$ and DMF molecule. ${ }^{6,24}$ The protective layer on the MAI- $\mathrm{PbI}_{2}-\mathrm{DMF}$ film prevent the DMF from escaping quickly. The restricted DMF induce solvent annealing effects during annealing process. Therefore, the solvent annealing effect response for the improved crystallization and large grains of $\mathrm{MAPbI}_{3}$ films annealed with protective layer. ${ }^{17}$ We also introduce optimized DMSO into $\mathrm{PbI}_{2} / \mathrm{DMF}$ solution ${ }^{6}$ by employing $\mathrm{PC}_{61} \mathrm{BM}$ as protective layer during annealing. The perovskite grains increase significantly, but it leads to many holes in perovskite film (see Fig. S3†), which have detrimental effects on the photovoltaic performance of PSCs. In order to confirm the university of the new annealing approach, we also use SpiroOMeTAD (without any additives) as protective layer to retard the escape of DMF molecule, it can also increase perovskite grain size and photovoltaic performance of corresponding devices due to solvent annealing effect (see Fig. $\mathrm{S} 4 \dagger$ ).

Fig. 3a is a cross-sectional SEM image of a complete PSCs, showing a geometric structure of the device containing fluorinedoped tin oxide (FTO)/compact- $\mathrm{TiO}_{2} /$ mesoporous- $\mathrm{TiO}_{2} / \mathrm{MAPbI}_{3} /$ Spiro-OMeTAD/Au. As shown in Fig. 3b, we achieved the best 

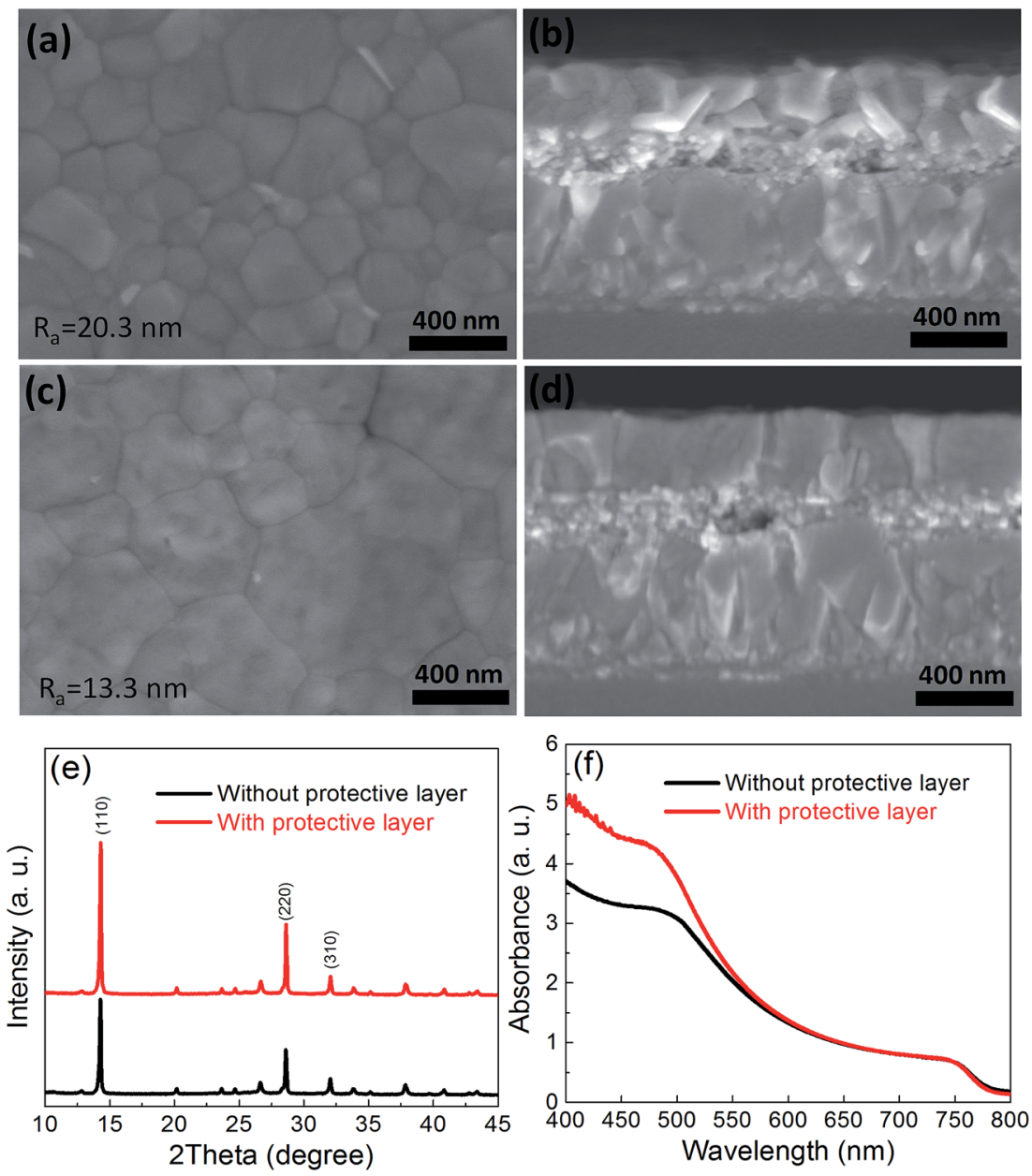

Fig. 2 Surface and cross-sectional SEM images of the perovskite films fabricated from different annealing approaches. (a) and (b) are without protective layer, (c) and (d) are with protective layer. (e) XRD patterns and (f) UV-Vis absorption spectra of the perovskite films fabricated from different annealing approaches.

photovoltaic performance of the PSCs fabricated from different annealing approaches by employing this structure. The PSCs annealed with protective layer exhibit an improved performance as follows: short-circuit $\left(J_{\mathrm{sc}}\right)=22.58 \mathrm{~mA} \mathrm{~cm}^{-2}$, open voltage $\left(V_{\mathrm{oc}}\right)$ $=1.03 \mathrm{~V}$, fill factor $(\mathrm{FF})=0.69$, power conversion efficiency $(\mathrm{PCE})=16.04 \%$. All of the photovoltaic parameters are better than those annealed without the protective layer $U_{\mathrm{sc}}=$ $\left.20.38 \mathrm{~mA} \mathrm{~cm}{ }^{-2}, V_{\mathrm{oc}}=0.95 \mathrm{~V}, \mathrm{FF}=0.66, \mathrm{PCE}=12.78 \%\right)$. The statistical results of the photovoltaic parameters extracted from a series of PSCs are shown in Fig. 4. The statistical results further confirm similar trend to the best PSCs fabricated from different annealing approaches. Fig. 3c shows the stabilized output at the maximum point of PSCs fabricate from different annealing approaches. It shows that the PSC annealed with a protective layer exhibits higher efficiency than that from annealed without the protective layer. It is noted that the stabilized output value at the maximum power point is lower than the value obtained from reverse scan, which is widely observed in PSCs with obvious hysteresis behaviors (see Fig. S5†). ${ }^{34-36}$ Incident photon to current efficiency (IPCE) spectra in Fig. 3d confirm that the PSCs annealed with protective layer exhibits higher quantum yield throughout the entire wavelength than that annealed without protective layer, which is consistent with the photovoltaic performance. The integrated $J_{\mathrm{sc}}$ obtained from IPCE spectra is lower than the value obtained from reverse scan. This discrepancy probably due to the slow response of $J_{\mathrm{sc}}$ (Fig. 3c), ${ }^{37}$ or the existence defective in $\mathrm{TIO}_{2},{ }^{38}$ or the mismatch in light spectra. ${ }^{39}$

Fig. $5 \mathrm{a}$ is Nyquist plots of impedance spectra for PSCs by employing a bias voltage of $0.9 \mathrm{~V}$ in dark. The simplified equivalent circuit is used to analysis the charge transportation and recombination in the PSCs. ${ }^{40-42}$ In this circuit, $R_{\mathrm{s}}$ is series resistance between two electrodes; charge transportation resistance $\left(R_{\mathrm{ct}}\right)$ in parallel with a chemical capacity $\left(\mathrm{CPE}_{\mathrm{ct}}\right)$ at high frequency, which ascribed to the perovskite-ETL and perovskite-HTL interface. The smaller value of $R_{\text {ct }}$ indicates better transportation of charges through those interface; recombination resistance $\left(R_{\text {rec }}\right)$ in parallel with a chemical capacity $\left(\mathrm{CPE}_{\text {rec }}\right)$ at low frequency, which reflects the recombination information in PSCs. The recombination resistance $\left(R_{\text {rec }}\right)$ is inversely related to the recombination rate of carriers, ${ }^{43}$ the larger value of $R_{\text {rec }}$ indicates the less charge recombination in 

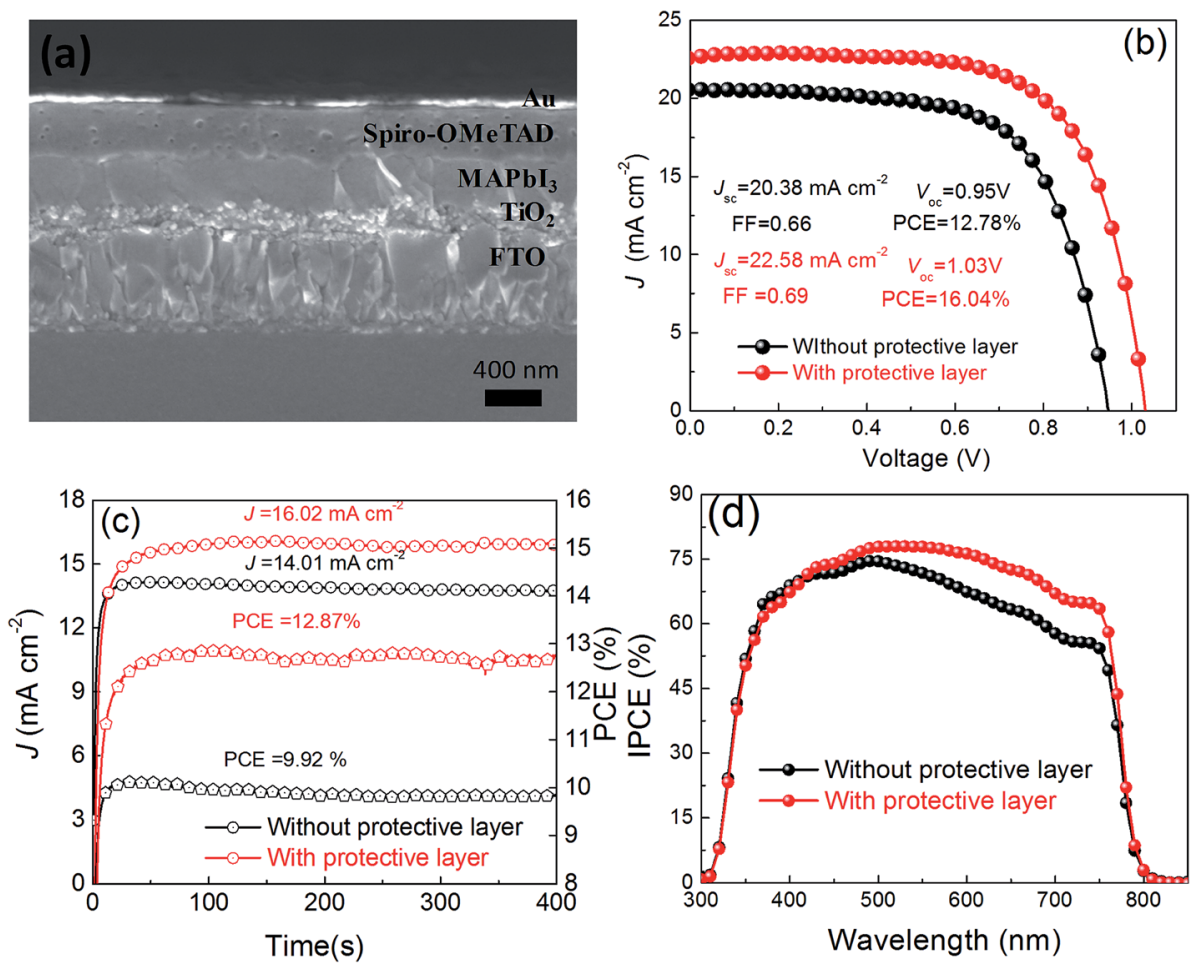

Fig. 3 (a) Typical cross-sectional SEM images of a complete PSC fabricated with a protective layer during the annealing process. (b) J-V curves of the best PSCs fabricated from different annealing approaches. (c) The stabilized output at the maximum power point. (d) Typical IPCE spectra of the PSCs solar cells fabricated with and without protective layers.

PSCs. The $R_{\mathrm{ct}}$ of PSCs fabricated by employing protective layer during annealing process is $133.4 \Omega$, which is lower than the value 167.3 $\Omega$ obtained from the PSCs fabricated without protective layer. The reduced charge transportation resistance indicates more efficient charge transportation of carries in the whole devices. Meanwhile, the recombination resistance $R_{\text {rec }}$ increased from 190.7 to $636.5 \Omega$ when the protective layer was introduced during annealing process, the larger value of $R_{\text {rec }}$ indicates the suppression of charge recombination due to the improvement of perovskite quality. The reduced recombination rate is beneficial to increase the FF and $V_{\mathrm{oc}}$ of PSCs (see Fig. 3b).

Fig. $5 \mathrm{~b}$ are the normalized steady photo-luminescence (PL) spectra for studying the effects of perovskite quality on charge transfer at the interface of $\mathrm{TiO}_{2}$ and perovskite film. The sample
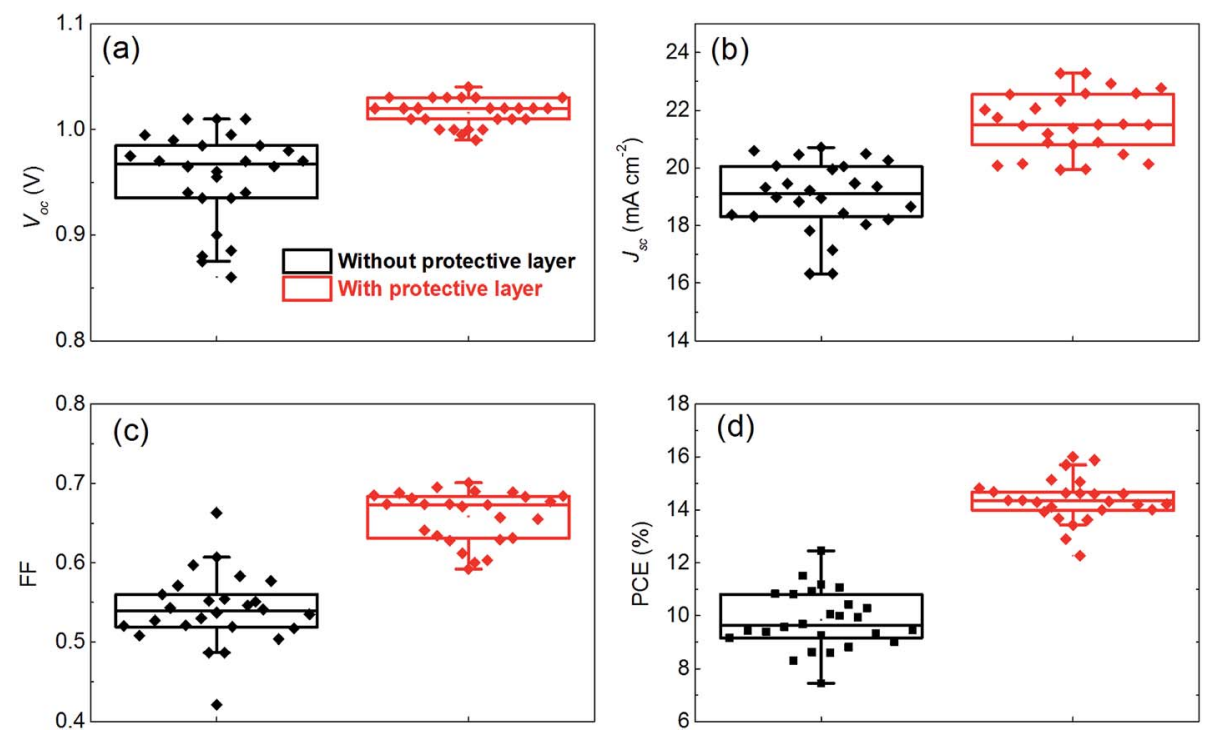

Fig. 4 Box chart of photovoltaic parameters of a series PSCs extracted from J-V curves. (a) $V_{\text {oc, }}$ (b) $J_{\text {sc, }}$ (c) FF, (d) PCE. 

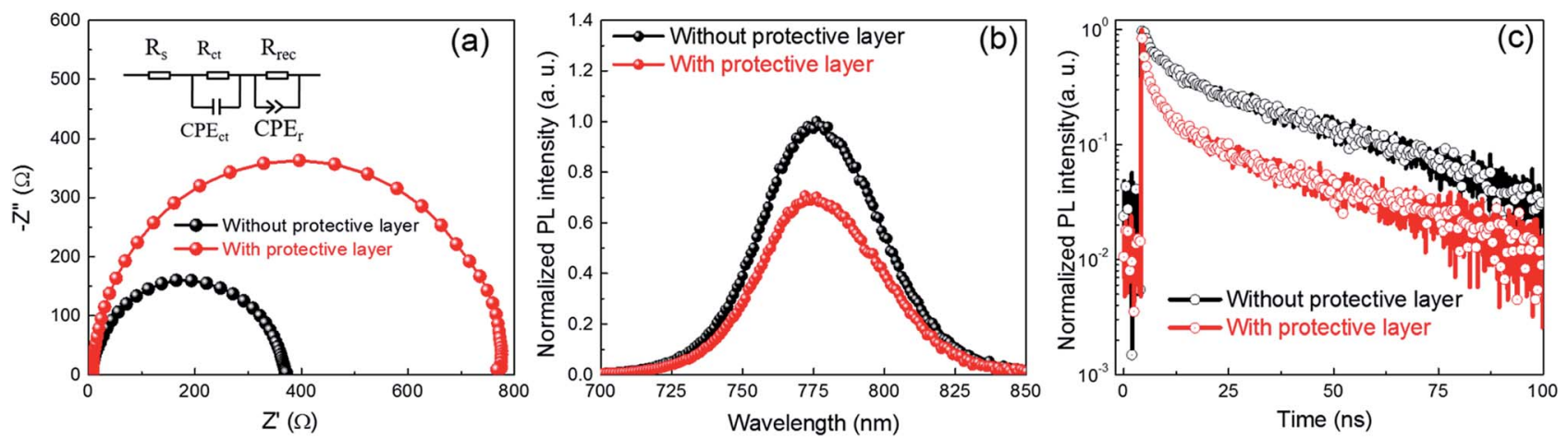

Fig. 5 (a) Nyquist plots of the PSCs measured in dark under a bias voltage of $0.9 \mathrm{~V}$. (b) Steady PL spectra of the MAPbl 3 film annealed by different approaches. (c) Time resolved PL spectra and the fitting results by using bi-exponential decay.

for steady PL measurement is composed of $\mathrm{FTO} / \mathrm{TiO}_{2} / \mathrm{MAPbI}_{3}$. Fig. 5b shows that a characteristic peak at $776 \mathrm{~nm}$ related the intrinsic bandgap $(\sim 1.60 \mathrm{eV})$ of $\mathrm{MAPbI}_{3}$, which is consistent with the UV-Vis absorption spectra (see Fig. 2f). As comparison with the samples annealed without protective layer, the PL intensity of sampled annealed with protective layer deceases significantly, which indicates more efficient charge injection from $\mathrm{MAPbI}_{3}$ to $\mathrm{TiO}_{2}$ layers. ${ }^{\mathbf{4 4}, 45}$ The efficient charge extraction ability can be ascribed to the column-like grains (see Fig. 2d) and improved grain orientation (see Fig. 2e). The improved charge extraction ability and absorption ability in the range from 400 to $800 \mathrm{~nm}$ (see Fig. 2f) should be responsible for the increased $J_{\mathrm{sc}}$ obtained from PSCs (see Fig. $3 \mathrm{~b}$ and $4 \mathrm{~b}$ ). Fig. $5 \mathrm{c}$ shows the time-resolved PL spectra of the perovskite film deposited on $\mathrm{TiO}_{2}$ substrates. The dynamic PL can be well fitted by a bi-exponential equation:

$$
I(t)=A_{1} \mathrm{e}^{-t / \tau_{1}}+A_{2} \mathrm{e}^{-t / \tau_{2}}
$$

where $A_{1}$ and $A_{2}$ are the decay amplitude, $\tau_{1}$ and $\tau_{2}$ are the decay time, respectively. For the perovskite obtained without protective layer, $\tau_{1}=3.68 \mathrm{~ns}$, and $\tau_{2}=38.12 \mathrm{~ns}$ with the decay amplitudes $9.41 \%$ and $90.59 \%$, respectively. When the protective layer is employed during the annealing process, $\tau_{1}$ and $\tau_{2}$ reduce to $1.51 \mathrm{~ns}$ and $18.74 \mathrm{~ns}$ with the decay amplitudes of $19.26 \%$ and $80.74 \%$, respectively. The average PL decay time $\tau_{\text {ave }}$ can be calculated by the following formula. ${ }^{42,46}$

$$
\tau_{\mathrm{ave}}=\frac{\sum A_{i} \tau_{i}^{2}}{\sum A_{i} \tau_{i}}
$$

The average PL decay time reduced from $37.77 \mathrm{~ns}$ to $18.42 \mathrm{~ns}$ when the protective layer is employed during annealing process. The reduced $\tau_{\text {ave }}$ also demonstrated that the efficient transportation and fast injection into $\mathrm{TiO}_{2}$ from perovskite film. The improved transportation and injection ability of charge can be ascribed to the less grain boundary along the thickness direction (see Fig. 2b and d). The reduced PL decay time is beneficial to reduce the combination at the interface at perovskite-ETL interface, which should be responsible for improvement in FF and $V_{\mathrm{oc}}$ (see Fig. 3b). So higher $J_{\mathrm{sc}}$ of PSC fabricated with protective layer can be ascribed strong light harvest ability from 400 to $800 \mathrm{~nm}$ and fast injection of carriers. The higher $V_{\mathrm{oc}}$ and FF can be ascribed to lower recombination rate due to less grains boundary in perovskite.

\section{Conclusions}

In summary, the solvent embedded in the perovskite precursor film play a key role in fabricating high quality perovskite films. It implies a solvent annealing effect during annealing of perovskite precursor film. We strengthen the intrinsic solvent annealing effects by pre-depositing a protective layer on the perovskite precursor film to retard the escape of solvent. The restricted solvent induces an obvious solvent annealing effect during annealing process. The strengthened solvent annealing effects lead to formation of high quality perovskite film with large grains and excellent grain orientation. The improved quality of perovskite film leads to efficient carrier transformation and injection, low recombination in devices. As a result, the performance of perovskite solar cells fabricated from corresponding perovskite film enhanced significantly. This work provides a facial method to obtain large grains by making use of self-embedded solvent in perovskite precursor film.

\section{Conflicts of interest}

The authors declare no competing financial interest.

\section{Acknowledgements}

This work was financially supported by Shenzhen Jiawei Photovoltaic Lighting Co. Ltd., and Tsinghua University Initiative Scientific Research Program (20161080165).

\section{References}

1 A. Kojima, K. Teshima, Y. Shirai and T. Miyasaka, J. Am. Chem. Soc., 2009, 131, 6050-6051. 
2 W. S. Yang, B. W. Park, E. H. Jung, N. J. Jeon, Y. C. Kim, D. U. Lee, S. S. Shin, J. Seo, E. K. Kim, J. H. Noh and S. I. Seok, Science, 2017, 356, 1376-1379.

3 D. Bi, J. Luo, F. Zhang, A. Magrez, E. N. Athanasopoulou, A. Hagfeldt and M. Grätzel, ChemSusChem, 2017, 10, 16241630.

4 X. B. Cao, L. L. Zhi, Y. H. Li, F. Fang, X. Cui, Y. W. Yao, L. J. Ci, K. X. Ding and J. Q. Wei, J. Mater. Chem. C, 2017, 5, 74587464.

5 Y. Zhang, L. Tan, Q. Fu, L. Chen, T. Ji, X. Hu and Y. Chen, Chem. Commun., 2016, 52(5), 674-5677.

6 X. B. Cao, C. L. Li, L. L. Zhi, Y. H. Li, X. Cui, Y. W. Yao, L. J. Ci and J. Q. Wei, J. Mater. Chem. A, 2017, 5, 8416-8422.

7 C. G. Wu, C. H. Chiang, Z. L. Tseng, M. K. Nazeeruddin, A. Hagfeldt and M. Grätzel, Energy Environ. Sci., 2015, 8, 2725-2733.

8 X. B. Cao, C. L. Li, Y. H. Li, F. Fang, X. Cui, Y. W. Yao and J. Q. Wei, Nanoscale, 2016, 8, 19804-19810.

9 N. J. Jeon, J. H. Noh, W. S. Yang, Y. C. Kim, S. C. Ryu, J. W. Seo and S. I. Seok, Nature, 2015, 517, 476-480.

10 H. D. Kim, H. Ohkita, H. Benten and S. Ito, Adv. Mater., 2016, 28, 917-922.

11 C. H. Chiang and C. G. Wu, ChemSusChem, 2016, 9, 26662672.

12 W. Nie, H. Tsai, R. Asadpour, J. C. Blancon, A. J. Neukirch, G. Gupta, J. J. Crochet, M. Chhowalla, S. Tretiak, M. A. Alam, H. L. Wang and A. D. Mohite, Science, 2015, 347, 522-525.

13 M. Xiao, F. Huang, W. Huang, Y. Dkhissi, Y. Zhu, J. Etheridge, A. Gray-Weale, U. Bach, Y. B. Cheng and L. Spiccia, Angew. Chem., Int. Ed., 2014, 53, 9898-9903.

14 M. Yang, T. Zhang, P. Schulz, Z. Li, G. Li, D. H. Kim, N. Guo, J. J. Berry, K. Zhu and Y. Zhao, Nat. Commun., 2016, 7, 12305.

15 S. Bag and M. F. Durstock, ACS Appl. Mater. Interfaces, 2016, 8, 5053-5057.

16 M. K. Kim, T. Jeon, H. I. Park, J. M. Lee, S. A. Nam and S. O. Kim, CrystEngComm, 2016, 18, 6090-6095.

17 Z. Xiao, Q. Dong, C. Bi, Y. Shao, Y. Yuan and J. Huang, Adv. Mater., 2014, 26, 6503-6509.

18 D. Liu, L. Wu, C. Li, S. Ren, J. Zhang, W. Li and L. Feng, ACS Appl. Mater. Interfaces, 2015, 7, 16330-16337.

19 J. Liu, C. Gao, X. He, Q. Ye, L. Ouyang, D. Zhuang, C. Liao, J. Mei and W. Lau, ACS Appl. Mater. Interfaces, 2015, 7, 24008-24015.

20 Y. Numata, A. Kogo, Y. Udagawa, H. Kunugita, K. Ema, Y. Sanehira and T. Miyasaka, ACS Appl. Mater. Interfaces, 2017, 9, 18739-18747.

21 N. J. Jeon, J. H. Noh, Y. C. Kim, W. S. Yang, S. Ryu and S. I. Seok, Nat. Mater., 2014, 13, 897-903.

22 Y. Rong, S. Venkatesan, R. Guo, Y. Wang, J. Bao, W. Li, Z. Fan and Y. Yao, Nanoscale, 2016, 8, 12892-12899.

23 Y. Rong, Z. Tang, Y. Zhao, X. Zhong, S. Venkatesan, H. Graham, M. Patton, Y. Jing, A. M. Guloy and Y. Yao, Nanoscale, 2015, 7, 10595-10599.
24 X. B. Cao, Y. H. Li, F. Fang, X. Cui, Y. W. Yao and J. Q. Wei, RSC Adv., 2016, 6, 70925-70931.

25 X. Cao, L. Zhi, Y. Li, F. Fang, X. Cui, Y. Yao, L. Ci, K. Ding and J. Wei, ACS Appl. Mater. Interfaces, 2017, 9, 32868-32875.

26 H. Zheng, W. Wang, S. Yang, Y. Liu and J. Sun, RSC Adv., 2016, 6, 1611-1617.

27 S. Bae, S. J. Han, T. J. Shin and W. H. Jo, J. Mater. Chem. A, 2015, 3, 23964-23972.

28 M. Long, T. Zhang, Y. Chai, C. F. Ng, T. C. W. Mak, J. Xu and K. Yan, Nat. Commun., 2016, 7, 13503.

29 J. Xu, A. Buin, A. H. Ip, O. Voznyy, R. Comin, M. Yuan, S. Jeon, Z. Ning, J. J. McDowell, P. Kanjanaboos, J. P. Sun, X. Lan, L. N. Quan, D. H. Kim, I. G. Hill, P. Maksymovych and E. H. Sargent, Nat. Commun., 2015, 6, 7081.

30 N. D. Marco, H. Zhou, Q. Chen, P. Sun, Z. Liu, L. Meng, E.-P. Yao, Y. Liu, A. Schiffer and Y. Yang, Nano Lett., 2016, 16, 1009-1016.

31 Y. Wang, J. Li, Q. Li, W. Zhu, T. Yu, X. Chen, L. Yin, Y. Zhou, X. Wang and Z. Zou, Chem. Commun., 2017, 53, 5032-5035.

32 A. R. M. Yusoff and M. K. Nazeeruddin, J. Phys. Chem. Lett., 2016, 7, 851-866.

33 F. Hao, C. C. Stoumpos, P. Guo, N. Zhou, T. J. Marks, R. P. H. Chang and G. Kanatzidis, J. Am. Chem. Soc., 2015, 137, 11445-11452.

34 Y. Zhang, M. Liu, G. E. Eperon, T. C. Leijtens, D. McMeekin, M. Saliba, W. Zhang, M. Bastiani, A. Petrozza, L. M. Herz, M. B. Johnston, H. Lin and H. J. Snaith, Mater. Horiz., 2015, 2, 315-322.

35 N. K. Noel, A. Abate, S. D. Stranks, E. S. Parrott, V. M. Burlakov, A. Goriely and H. J. Snaith, ACS Nano, 2014, 8, 9815-9821.

36 S. Bai, N. Sakai, W. Zhang, Z. Wang, J. T. W. Wang, F. Gao and H. J. Snaith, Chem. Mater., 2017, 29, 462-473.

37 J. W. Lee, H. S. Kim and N. G. Park, Acc. Chem. Res., 2016, 49, 311-319.

38 L. Li, Y. Chen, Z. Liu, Q. Chen, X. Wang and H. Zhou, Adv. Mater., 2016, 28, 9862-9868.

39 C. Sun, Y. Guo, B. Fang, J. Yang, B. Qin, H. Duan, Y. Chen, H. Li and H. Liu, J. Phys. Chem. C, 2016, 120, 12980-12988.

40 S. Wang, W. Dong, X. Fang, Q. Zhang, S. Zhou, Z. Deng, R. Tao, J. Shao, R. Xia, C. Song, L. Hu and J. Zhu, Nanoscale, 2016, 8, 6600-6608.

41 D. Liu, M. K. Gangishetty and T. L. Kelly, J. Mater. Chem. A, 2014, 2, 19873-19881.

42 D. Yang, X. Zhou, R. Yang, Z. Yang, W. Yu, X. Wang, C. Li, S. Liu and R. P. H. Chang, Energy Environ. Sci., 2016, 9, 3071-3078.

43 J. A. Christians, R. C. M. Fung and P. V. Kamat, J. Am. Chem. Soc., 2014, 136, 758-764.

44 H. S. Ko, J. W. Lee and N. G. Park, J. Mater. Chem. A, 2015, 3, 8808-8815.

45 Q. Jiang, L. Zhang, H. Wang, X. Yang, J. Meng, H. Liu, Z. Yin, J. Wu, X. Zhang and J. You, Nat. Energy, 2016, 2, 16117.

46 D. Yang, R. Yang, X. Ren, X. Zhu, Z. Yang, C. Li and S. Liu, Adv. Mater., 2016, 28, 5206-5213. 